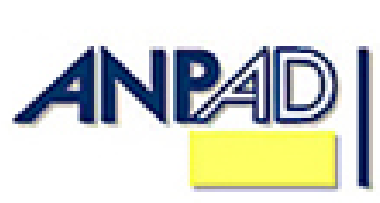

Disponível em

http://www.anpad.org.br/rac

RAC, Rio de Janeiro, v. 16, n. 3, art. 2,

pp. 360-380, Maio/Jun. 2012

$(\mathrm{coc}$ EY-No

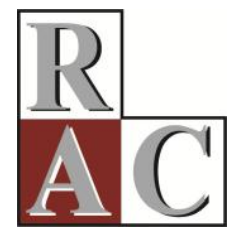

\title{
Vantagem Competitiva e sua Relação com o Desempenho - uma Abordagem Baseada em Valor
}

\author{
Competitive Advantage and Performance: a Value Creation Approach
}

Renata Peregrino de Brito *

E-mail: renatap.brito@gmail.com

Ibmec - RJ

Rio de Janeiro, RJ, Brasil.

Luiz Artur Ledur Brito

E-mail: Luiz.brito@fgv.br

Fundação Getúlio Vargas- EAESP/FGV

São Paulo, SP, Brasil.

* Endereço: Renata Peregrino de Brito

Av. Pres. Wilson, 118, Rio de Janeiro/RJ, 20030-020.

Copyright (C) 2012 RAC. Todos os direitos, até mesmo de tradução, são reservados. É permitido citar parte de artigos sem autorização prévia, desde que seja identificada a fonte. 


\title{
Resumo
}

Este ensaio teórico discute o conceito de vantagem competitiva identificando os desafios da área de estratégia em obter uma definição precisa, operacionalizável e que relacione o conceito com o desempenho das empresas. Inicia-se pela consolidação e pela análise dos principais trabalhos na área, em que explicitamos as lacunas e a falta de diferenciação entre os construtos de vantagem competitiva e desempenho. $\mathrm{O}$ artigo traz uma definição baseada na criação de valor superior, sendo esse delimitado pela diferença entre a disposição a pagar dos clientes e o custo de oportunidade dos fornecedores. Tal definição sugere que o valor criado pode ser divido em três partes: o valor apropriado pela empresa, o excedente dos clientes e o excedente dos fornecedores. Cada uma das partes traz implicações para o desempenho da empresa, sendo que o valor apropriado revela o lucro desta, e o valor não apropriado pode se traduzir em crescimento e em desempenho operacional. No tocante à operacionalização da vantagem competitiva, discutimos aspectos relativos à comparação entre empresas e à temporalidade de observação do evento. Como decorrências desse ensaio, está o estudo da lógica causal entre vantagem competitiva e desempenho, e a constatação de que o estudo da vantagem competitiva por meio de variável única é impreciso e não mede a extensão dos resultados das decisões estratégicas.

Palavras-chave: vantagem competitiva; criação de valor; apropriação de valor; desempenho.

\begin{abstract}
This theoretical paper discusses the concept of competitive advantage by identifying the challenges the field of business strategy faces in obtaining a precise and operational definition that links the concept to firm performance. Starting by the analysis and consolidation of literature in the area, we explicitly display the gaps and lack of differentiation between the constructs of competitive advantage and performance. This paper provides a definition based on the superior creation of value, with value defined as the difference between customers' willingness to pay and suppliers' opportunity cost. This definition suggests that the value created can be divided into three parts: the value appropriated by the firm, the customers' surplus, and the suppliers' surplus. Each part has implications for firm performance: while the appropriated value reveals the profit level, the value not appropriated can be translated into growth and operational performance. Regarding the operationalization of competitive advantage, we discuss aspects related to comparison between firms and the temporal observation of the event. As the main result of this discussion, we explore the causal logic between competitive advantage and performance and conclude that competitive advantage cannot be depicted by the observation of a unique variable, as it is imprecise and does not measure the extent of impacts strategic decisions have.
\end{abstract}

Key words: competitive advantage; value creation; value appropriation; performance. 


\section{Introdução}

A busca por vantagem competitiva está presente em boa parte dos estudos sobre estratégia, assim como nas orientações das decisões empresariais. Vantagem competitiva é tida como a principal hipótese para explicar o desempenho superior das empresas, e, assim, passa a representar o objetivo fundamental da gestão estratégica (Powell, 2001; South, 1981). Enquanto autores debatem as origens, os determinantes e a sustentabilidade da vantagem competitiva, de tão usual o termo passa a ser empregado de forma autoexplicativa (Adegbesan, 2009; Caves, 1984; Reed \& DeFillippi, 1990; Teece, Pisano, \& Shuen, 1997). No entanto, um olhar mais cuidadoso revela que, por mais ubíquo que seja, vantagem competitiva está sujeita a diferentes interpretações, o que acarreta dificuldade de mensuração e falseabilização do conceito (Arend, 2003; Rumelt, 2003).

Nos estudos empíricos, um problema comum está no design operacional do construto e na falta de clareza e diferenciação entre os conceitos de vantagem competitiva e de desempenho. Powell (2001) argumenta que, se a vantagem competitiva é definida como desempenho superior, ou se a relação entre os construtos sempre existir de forma direta, tal vantagem torna-se dispensável. Portanto, há necessidade de definir e investigar a lógica da relação de causa e efeito entre os construtos.

No debate teórico, nem a definição nem a delimitação de vantagem competitiva são um consenso. Entre outros, Rumelt (2003) reconhece a crescente convergência para o conceito de criação de valor, que, no entanto, também apresenta diferentes interpretações entre autores e áreas do conhecimento. Afinal, criação de valor é um conceito amplo que pode ser visto em perspectivas distintas, seja pelos clientes, empregados, fornecedores, acionistas ou demais stakeholders da empresa (Lepak, Smith, \& Taylor, 2007). Portanto, ao referenciar o conceito de criação de valor, faz-se necessária uma delimitação de escopo e referência utilizados.

No tocante às pesquisas empíricas, boa parte dos estudos limita-se a modelar métricas de desempenho que não estão necessariamente relacionadas à vantagem competitiva (Coff \& Lee, 2003; Lippman \& Rumelt, 2003a). Comum nos estudos de estratégia, a análise da lucratividade e dos índices de rentabilidade revela apenas o domínio da apropriação de valor pela empresa, definido pelos limites de valor de troca (preço e custo). Já o intervalo da criação de valor inclui os limites de valor de uso, nas fronteiras de disposição a pagar e custo de oportunidade (Bowman \& Ambrosini, 2000; Brandenburger \& Stuart, 1996). Sob a abrangência da criação de valor, estão os resultados da interação e do desenvolvimento de recursos e capacidades entre a empresa e seus parceiros comerciais. Esses temas importantes e atuais no debate de estratégia podem ser negligenciados pelo uso exclusivo de métricas de lucratividade.

Ao definir e delimitar o construto, é preciso abarcar o amplo escopo de estudo de estratégia empresarial e as interações internas e externas à empresa. Assim, na interação com clientes, a criação de valor traz implicações para a fidelização destes e para a repetição da compra (Newbert, 2008; Priem, 2007). Ao longo da cadeia de suprimentos, a criação de valor pode trazer resultados de qualidade e eficiência à empresa, além da diminuição de custos (Dyer \& Singh, 1998). Ademais, nas interações internas e externas à empresa, o valor criado traz resultados de desenvolvimento de tecnologia e inovação (Inkpen \& Tsang, 2005; Thomke \& Hippel, 2002). Portanto, a criação de valor envolve um escopo amplo e permite comparar a competitividade das empresas, incluindo outros resultados de desempenho que apenas lucratividade.

Assim sendo, este ensaio teórico tem como objetivos: consolidar o debate sobre vantagem competitiva, identificando os problemas atuais na acepção do conceito e propondo uma definição que responda a esses desafios; e teorizar como a vantagem competitiva se relaciona com o desempenho da empresa.

Para tanto, inicialmente faz-se uma revisão da evolução do conceito de vantagem competitiva e da convergência em direção à noção de criação de valor, na qual são discutidas as diferenças entre as diversas abordagens. Na seção seguinte, apresenta-se o conceito de criação de valor, suas fronteiras e 
os limites da apropriação de valor. Na sequência, é apresentada e discutida a proposta de definição de vantagem competitiva, seu escopo e seu contexto de aplicação. Segue a discussão sobre a distribuição do valor ao longo da cadeia, assim como os seus efeitos sobre o desempenho da empresa. Por fim, o artigo conclui sintetizando as contribuições e as implicações gerenciais e para a pesquisa.

\section{A Evolução do Conceito de Vantagem Competitiva}

A noção de vantagem competitiva, ou a possibilidade de uma empresa se destacar e superar o mercado, pode ser identificada já no modelo teórico de competição monopolística de Chamberlin (1933). Inicialmente, o termo aparece de forma eventual. Ansoff $(1965$, p. 93) se refere a ele como a uma "posição concorrencial" vantajosa obtida pelo ajuste de produtos a mercados. No final da década de 70, e inspirada pelo avanço da indústria japonesa, a preocupação com a competição ganha importância num ambiente dominado pelo planejamento estratégico, e o termo vantagem competitiva aparece em várias publicações, ainda com uma acepção de posição concorrencial, sem uma definição precisa (Allen, 1978; Morrisson \& Lee, 1979; Ohmae, 1978). A década de 80 marca uma mudança de foco da área de estratégia, saindo do planejamento e do portfólio para uma maior ênfase na competição, e, nesse contexto, o termo vantagem competitiva torna-se mais frequente (Ghemawat, 2002). Várias publicações enfatizam essa mudança, e a noção de vantagem em relação aos competidores passa a ser dominante (Gluck, Kaufman, \& Walleck, 1980; Hayes \& Wheelwright, 1984; South, 1981).

A publicação do livro de Michael Porter (1985), Vantagem Competitiva, consagra o emprego do termo entre consultores e acadêmicos. De maneira geral, a vantagem competitiva assume uma posição central no pensamento estratégico como principal objetivo de pesquisa (Reed \& DeFillippi, 1990). Vantagem competitiva passa a ser empregada com um significado genérico, por vezes associada a um conceito indefinido de valor (Barney, 1991; Porter, 1985), e muitas vezes identificada com o desempenho financeiro superior (Amit \& Schoemaker, 1993; Peteraf, 1993; Vasconcelos \& Brito, 2004). A Tabela 1 identifica algumas das principais definições usadas na literatura ilustrando a falta de consenso e imprecisão de um conceito tão relevante para a área.

Tabela 1

Definições para Vantagem Competitiva

\section{Definição}

"Trata-se da vantagem competitiva. Procura identificar propriedades específicas e combinações individuais de produtos e mercados que dão à empresa uma forte posição concorrencial”. (Ansoff, 1965, p. 93).

"Vantagens competitivas, isto é, posições únicas que uma organização desenvolve vis-à-vis seus competidores por meio de seu padrão de implantação de recursos e / ou escopo de decisões”. (Hofer \& Schendel, 1978, p. 25, tradução nossa).

"Em suma, vantagem competitiva é aquela que oferece a oportunidade de rentabilidade sustentada em relação aos concorrentes, em vez de uma circunstância na qual os lucros são capturados por empresas com posições semelhantes lutando por volume e market-share”. (South, 1981, p. 17, tradução nossa).

"Vantagem competitiva surge, fundamentalmente, do valor que uma empresa é capaz de criar para seus compradores, valor este que excede o custo da empresa em criá-lo. Valor é o que os compradores estão dispostos a pagar, e valor superior provém da oferta de preços inferiores aos dos concorrentes para benefícios equivalentes ou o fornecimento de benefícios únicos que mais do que compensam um preço superior. Existem dois tipos básicos de vantagem competitiva: liderança de custos e diferenciação". (Porter, 1985, p. 3, tradução nossa). 


\section{Tabela 1 (continuação)}

\begin{tabular}{l}
\hline Definição \\
\hline "Diz-se que uma empresa tem vantagem competitiva sustentada quando ela está implementando uma estratégia \\
de criação de valor que não está sendo implementada simultaneamente por algum dos concorrentes atuais ou \\
potenciais, e quando esses concorrentes são incapazes de duplicar os benefícios desta estratégia". (Barney, 1991, \\
p. 102, tradução nossa).
\end{tabular}

"Para os gestores, o desafio é identificar, desenvolver, proteger e alocar os recursos e capacidades de modo que forneçam à empresa uma vantagem competitiva sustentável e, assim, um retorno sobre capital superior". (Amit \& Shoemaker, 1993, p. 33, tradução nossa).

"Uma vantagem competitiva sustentável ou sustentada ocorre quando uma empresa implementa uma estratégia de criação de valor da qual outras empresas são incapazes de duplicar os benefícios ou os mesmos são custosos de imitar”. (Hitt, Ireland, \& Hoskisson, 1999, p. 5, tradução nossa).

"Em resumo, propomos a seguinte definição de vantagem competitiva: o diferencial entre dois concorrentes em qualquer dimensão concebível, que permita a um criar mais valor para o cliente do que ao outro". (Ma, 2000, p. 18, tradução nossa).

"Quando duas ou mais empresas competem no mesmo mercado, uma empresa possui uma vantagem competitiva sobre seus rivais quando ganha (ou tem o potencial para ganhar) uma taxa do lucro persistentemente mais alta". (Grant, 2002, p. 227, tradução nossa).

"Nós seguimos Peteraf (1993) ao pensar na relevante variável dependente, isto é, a vantagem competitiva sustentada como diferencial estritamente positivo de lucro em excesso aos custos de oportunidade (incluindo o custo de capital) que são mantidos em equilíbrio, no qual os diferenciais mais relevantes podem estar entre indústrias bem como intraindústria”. (N. J. Foss \& Knudsen, 2003, p. 291, tradução nossa).

"Uma empresa tem uma vantagem competitiva se for capaz de criar mais valor econômico do que o concorrente marginal (breakeven) em seu mercado de produto.... O valor econômico criado por uma empresa no decurso da prestação de um bem ou um serviço é a diferença entre os benefícios percebidos pelos compradores ao adquirirem o bem e o custo econômico para a empresa". (Peteraf \& Barney, 2003, p. 314, tradução nossa).

"Este artigo propõe que a vantagem competitiva seja concebida como a influência líquida de todos os fatores idiossincráticos da empresa sobre o seu desempenho durante um período determinado, excluídas as influências de outros fatores, como a indústria, os fatores temporais e o erro estatístico". (Vasconcelos \& Brito, 2004, p. 74).

"Diz-se que a empresa tem uma vantagem competitiva sobre a rival se ela tiver impulsionado um amplo espaço entre a disposição a pagar que gera entre seus compradores e os custos que incorre - na verdade, um espaço mais amplo do que o alcançado por seus concorrentes”. (Ghemawat \& Rivkin, 2006, p. 3, tradução nossa).

Um dos problemas mais graves está no tratamento de vantagem competitiva como desempenho financeiro superior. Isso porque os dois conceitos (vantagem competitiva e desempenho superior) passam a ser tratados como tendo uma relação causal direta e perfeita e são intercambiados com frequência. Esse tratamento leva a um questionamento da própria utilidade do conceito, que não adicionaria qualquer explicação para a variável dependente desempenho (Powell, 2001).

Em debates de fóruns relevantes na área de estratégia, como a divisão de Business Policy do Academy of Management e a Strategic Management Society, assim como na literatura, tem se configurado um avanço na direção de um consenso sobre a necessidade de precisão na definição. Coff (1999) foi o primeiro a apontar que vantagem competitiva pode não necessariamente corresponder a um desempenho superior, mostrando como a ideia de criação de valor para a definição de vantagem competitiva pode diferenciá-la do desempenho. Neste sentido, Rumelt (2003) identifica uma convergência em torno do conceito de criação valor superior aos concorrentes. Peteraf e Barney (2003), em artigo resposta a um questionamento de N. J. Foss e Knudsen (2003), no qual redefinem vantagem competitiva em termos de valor superior (ver Tabela 1) consolidando formulações anteriores e apoiando-se numa definição mais detalhada de valor econômico proposta inicialmente por Brandenburger e Stuart (1996). Vários autores, de forma paralela, passam a utilizar variações do conceito de valor econômico proposto por Brandenburger e Stuart (1996) para se referir tanto à vantagem competitiva como para explicar desempenho superior (Bowman \& Ambrosini, 2000; Coff, 
2010; Ghemawat \& Rivkin, 2006; Lippman \& Rumelt, 2003a, 2003b; Newbert, 2008). De maneira análoga, o modelo VPC (Value - Price - Cost), derivado de modelos de barganha, é objeto de um número especial do Strategic Management Journal (Hoopes, Madsen, \& Walker, 2003). O tema é recentemente retomado no painel sobre vantagem competitiva da conferência de 2010 do Academy of Management (Drnevich, Madsen, \& Newbert, 2010), com importantes indagações ainda em aberto: "Como resultado, várias questões importantes para o estudo da vantagem competitiva permanecem sem resposta, tais como: Como podemos efetivamente retratar, modelar e medi-la? Como as dinâmicas organizacional, competitiva e ambiental a afetam? Como ela se desenvolve?" (Drnevich et al., 2010, p. 2, tradução nossa).

Embora os sinais de convergência sejam evidentes, a definição de vantagem competitiva ainda tem desafios importantes. Um primeiro desafio está na delimitação do que é valor criado. Esse ponto é desenvolvido mais extensamente na próxima seção deste artigo. Um segundo desafio é estabelecer o referencial contra o qual se determina a vantagem. E o terceiro desafio é definir a dimensão de tempo do conceito. Seria vantagem competitiva um estado momentâneo, ou nos referimos a um intervalo de tempo? Tais desafios são também abordados nas seções subsequentes compondo a proposta de definição constitutiva do termo. Iniciamos pela revisão do conceito de valor criado que é utilizado nas seções seguintes.

\section{Definindo Valor}

A discussão de valor é frequentemente abordada em estratégia, cujo conceito econômico distingue-se entre valor de uso e valor de troca de bens e serviços (Ito, Hayashi, Gimenez, \& Fensterseifer, 2012). Segundo as principais linhas teóricas, valor é gerado nos processos de transformação dos recursos na cadeia de valor (Barney, 1991; Bowman \& Ambrosini, 2000; Porter, 1985), envolvendo vários atores ao longo da cadeia (Dyer \& Singh, 1998). Contudo, a discussão sobre valor deve distinguir ainda dois momentos diferentes e muitas vezes confundidos: a criação de valor e a apropriação de valor, conforme veremos.

\section{Criação de valor}

Buscando refinar a discussão de criação de valor, Brandenburger e Stuart (1996) definem o domínio da criação de valor pela empresa como a diferença entre o custo de oportunidade do fornecedor e a disposição a pagar pelo cliente. Ao delimitar o valor de uso, ambos os conceitos utilizados são teóricos e de difícil mensuração.

$\mathrm{Na}$ literatura de marketing, o conceito de disposição a pagar representa o valor monetário que inclui todo o benefício que o cliente aufere no uso ou consumo do produto (Krishna, 1991; Wertenbroch \& Skiera, 2002). A disposição para pagar é subjetiva e depende da percepção do cliente sobre os benefícios oferecidos, o que difere entre clientes e entre contextos competitivos (Lindgreen \& Wynstra, 2005). A subjetividade do julgamento de utilidade e valor aplica-se tanto na relação com consumidores finais como nas relações entre empresas e seus respectivos gestores. Somente é valorado aquilo que é percebido, e a interação empresa-cliente permite a formação de estratégias de criação de valor: a empresa pode gerar mais valor redirecionando sua oferta a diferentes mercados, promovendo o produto, alterando a forma e o local de exposição e atuando junto a formadores de opinião.

Na outra fronteira da criação de valor, o custo de oportunidade é dado como o valor mínimo pelo qual o fornecedor está disposto a vender seus serviços e seus produtos (Ghemawat \& Rivkin, 2006), abaixo do qual esse não mais aceitará transacionar (Brandenburger \& Stuart, 1996). De maneira análoga, esse limite também dependerá das circunstâncias de interação entre as partes envolvidas, assim como da oferta dos demais concorrentes. Assim sendo, o custo de oportunidade é indeterminável de forma absoluta, o valor a ele atribuído acaba por ser o resultado do processo de 
barganha sobre o valor já criado ou sobre a expectativa de valor no futuro (Lippman \& Rumelt, 2003a). O caso do desenvolvimento da rede de relacionamentos Facebook e as interações entre os criadores ilustram a diversidade de valoração entre parceiros de um mesmo projeto. Com diferentes expectativas de uso, abrangência e capacidade de comercialização no espaço virtual, cada parte tinha uma apreciação do potencial econômico do site de relacionamentos.

Considerando que o contexto das relações das empresas com seus parceiros traz implicações diretas para a criação de valor, as fronteiras definidas pela disposição a pagar e pelo custo de oportunidade são circunstanciais. A interação entre os parceiros gera oportunidades para arbitragem, na exploração de discrepâncias entre as percepções de valor. Portanto, ao se posicionar no mercado e ao escolher parceiros comerciais, a empresa negocia os limites da criação de valor.

A resultante de valor criado nos processos de coespecialização e complementaridade na combinação de recursos entre parceiros é superior à soma dos recursos individualmente aportados pelas partes (Adegbesan \& Higgins, 2011; Lippman \& Rumelt, 2003a). Seja o valor de um recurso $\mathrm{v}\left(\mathrm{R}_{1}\right)$ e outro recurso $\mathrm{v}\left(\mathrm{R}_{2}\right)$, a união dos recursos traz um excedente $(\mathrm{S})$ à simples soma dos mesmos. Portanto: $v\left(R_{1} \cup R_{2}\right)=v\left(R_{1}\right)+v\left(R_{2}\right)+S$ (Adegbesan \& Higgins, 2011, p. 189). A forma de combinação dos recursos e a escolha dos parceiros de transação influem tanto no montante de valor criado como na percepção de valor pelas partes, em que não são claros os limites e os direitos de propriedade (K. Foss \& Foss, 2005). Tal processo traz implicações para o processo de apropriação de valor, como vemos a seguir.

\section{Apropriação de valor}

Após o momento de criação, segue-se o processo de barganha entre os atores para apropriação do valor criado pelas partes. Como resultado desse processo, é definido o valor de troca: preço e custo, e, portanto, o lucro da empresa (Figura 1). Boa parte dos estudos e modelagens sobre o processo de barganha está fundamentada na teoria dos jogos, pela qual os atores vão buscar individualmente maximizar a apropriação de valor (Brandenburger \& Stuart, 1996). Já sob uma perspectiva colaborativa, os atores vão buscar uma partilha justa do valor criado (Harrison, Bosse, \& Phillips, 2010). E, em uma perspectiva mista, os atores vão assegurar que o processo de barganha e distribuição não obstrua os relacionamentos e a capacidade de criação de valor (Brandenburger \& Nalebuff, 1996; Dagnino \& Padula, 2002).

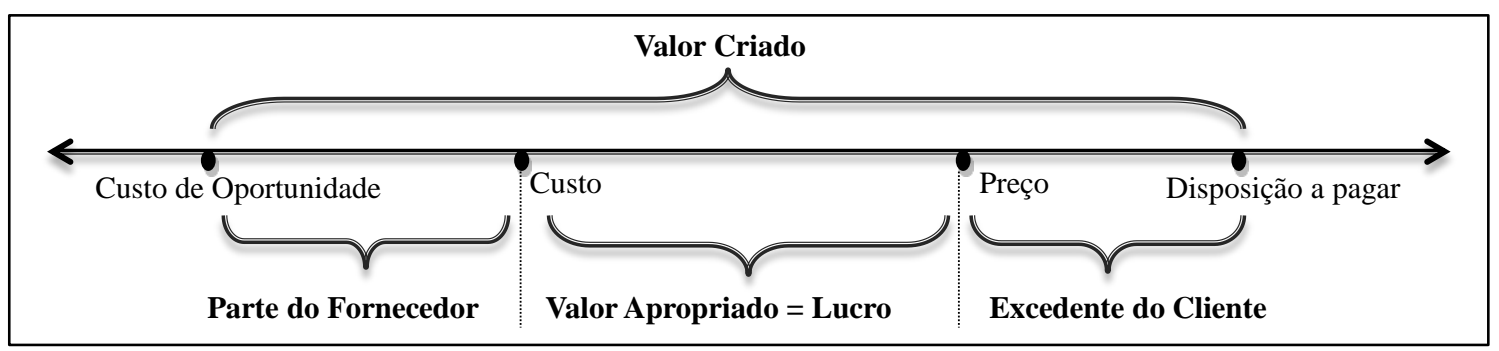

Figura 1. Valor Criado x Valor Apropriado.

Fonte: Adaptado de Brandenburger, A. M., \& Stuart, H. W. (1996). Value-based business strategy (p. 10). Journal of Economics \& Management Strategy, 5(1), 5-24. doi: 10.1111/j.1430-9134.1996.00005.x

Há ainda perspectivas mais amplas sobre a avaliação do valor criado, nas quais todos os stakeholders, sendo aqueles que afetam ou são afetados pela empresa, passam a ser inseridos (Freeman, 1984). Essa abordagem amplia os objetivos da empresa para além do foco no acionista, e considera o papel das mesmas na promoção de bem estar para a sociedade (Richard, Devinney, Yip, \& Johnson, 2009; Venkatraman \& Ramanujam, 1986). Sob essa visão, alguns estudos abordam stakeholders como participantes do processo de criação de valor da partilha do valor criado (Buysse \& Verbeke, 2003; Harrison et al., 2010; Hillman \& Keim, 2001).

O conceito de criação de valor permite incorporar mais de uma perspectiva do debate de estratégia empresarial. Seja pela visão do acionista, pela busca de alianças ou pelo estudo da 
legitimidade da atuação empresarial, o conceito de criação de valor abarca os vários interesses e pode ancorar o debate de competitividade de maneira mais ampla. Assim sendo, passamos a propor uma definição para vantagem competitiva.

\section{A Proposta de Definição de Vantagem Competitiva}

Definida como criação de valor superior aos concorrentes diretos, vantagem competitiva compreende o escopo entre a disposição a pagar e o custo de oportunidade da empresa. Em termos de abrangência, esse intervalo integra vários interesses de pesquisa em estratégia, seja a discussão sobre a cadeia de valor (Besanko, Dranove, Shanley, \& Schaefer, 1996; Ghemawat \& Rivkin, 2006; Porter, 1985), seja o debate sobre o desenvolvimento de recursos e capacidades (Blyler \& Coff, 2003; Dyer \& Singh, 1998; Hansen, Hoskisson, \& Barney, 2008).

As empresas capazes de criar mais valor são aquelas que apresentam uma vantagem competitiva frente a seus competidores. A superioridade na criação de valor propicia capacidade de manobra e estratégias de competitividade, movimentos esses que são ilustrados pela Figura 2, pelas estratégias genéricas de baixo custo e diferenciação (Ghemawat \& Rivkin, 2006).

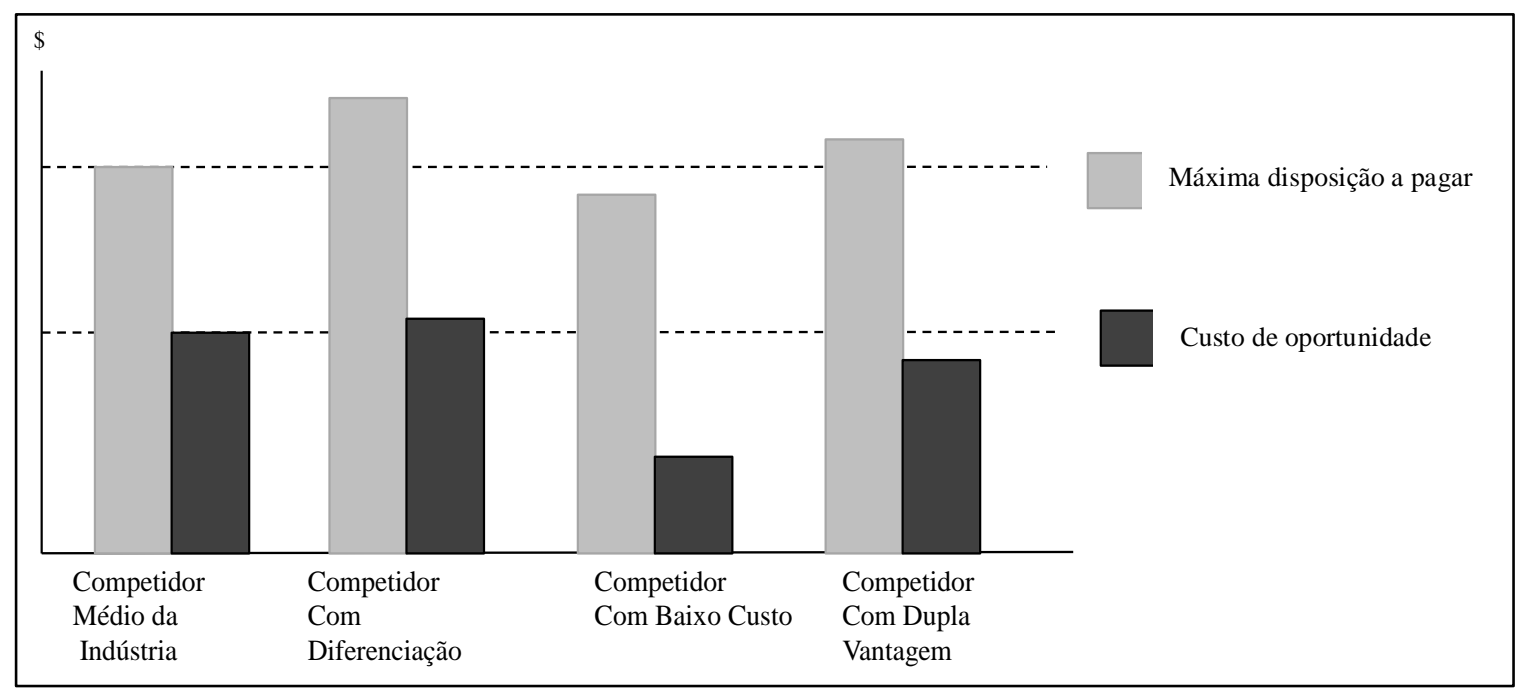

Figura 2. Tipos de Vantagem Competitiva.

Fonte: Ghemawat, P., \& Rivkin, J. W. (2006). Creating competitive advantage (p. 8). Boston: Harvard Business School Note.

Sendo vantagem competitiva um conceito relativo, passamos agora a explorar como se pode comparar a criação de valor entre empresas.

\section{$O$ valor adicionado pelas empresas}

Afirmar que a empresa cria valor não necessariamente confere vantagem à mesma, já que somente pela comparação entre empresas podemos auferir a vantagem competitiva. Dentro de um mesmo setor industrial, ou de um mercado consumidor específico, a criação de valor entre empresas concorrentes pode ser confrontada para que se perceba o valor adicionado. $\mathrm{O}$ valor criado, como uma medida absoluta, é relativizado para o contexto competitivo, e torna-se uma medida relativa de vantagem.

A medida de valor adicionado, ou added value, é também um termo amplamente empregado na literatura de economia e marketing, com definições distintas (Davis \& Kay, 1990; Wood, 1996). Em estratégia, Brandenburger e Stuart (1996, p. 6, tradução nossa) propõem que o valor adicionado seja 
medido como "o valor criado por todos os concorrentes na cadeia vertical menos o valor criado por todos os concorrentes exceto o participante em questão". Segundo essa definição, o valor adicionado depende do ambiente competitivo e do grupo de competidores selecionados que, de forma mais precisa, seriam apenas os competidores diretos da empresa (Chatain, 2011).

Peteraf e Barney (2003) propõem uma comparação entre empresas na sua capacidade de criar mais valor em relação ao competidor marginal no mercado de produtos. Assim, os autores reconhecem que "Isso implica que algumas ou mesmo várias empresas em uma dada indústria possam ter vantagem competitiva e sugere que haja diferentes caminhos para a vantagem competitiva" (Peteraf \& Barney, 2003, p. 315, tradução nossa). Portanto, para os autores, vantagem competitiva não se restringe ao melhor competidor apenas, mas a todas as empresas que criarem mais valor do que o competidor que opera acima do ponto de equilíbrio.

Se, por um lado, a definição de Brandenburger e Stuart (1996) reconhece somente o líder de criação de valor em cada contexto competitivo, por outro, a proposta de Peteraf e Barney (2003) pode atribuir vantagem competitiva a empresas que criam valor em níveis inferiores à média da indústria. Entre as duas propostas, há uma questão de contexto no qual o valor é apurado. Embora seja possível admitir que dentro de uma mesma indústria mais de uma empresa tenha vantagem competitiva, afinal elas podem estar inseridas em diferentes contextos competitivos, há que se reconhecer um líder dentro de cada contexto. Assim sendo, a vantagem competitiva passa a ser uma medida de dispersão em torno de uma média ou mediana de uma indústria. Sendo o valor adicionado uma medida da superioridade da empresa em relação aos seus competidores diretos, a variância desse valor revela a volatilidade dessa posição e a capacidade de sustentação da mesma.

\section{Vantagem competitiva no tempo}

Qual o tempo necessário para que se configure uma vantagem competitiva? Afinal, se a vantagem competitiva é um evento frágil ou efêmero, não há motivos para estudá-la. De fato, vários autores e estudos questionam não só a existência de vantagem competitiva, como a sua manutenção no tempo (D’Aveni, Dagnino, \& Smith, 2010; Williamson, 1991).

A vantagem competitiva sustentada, ou sustained competitive advantage, foi introduzida como aquela que rende um desempenho superior em longo prazo (Porter, 1985). Contudo, a sustentabilidade a que se refere o termo não diz respeito aos efeitos da vantagem competitiva sobre o desempenho, mas à capacidade da manutenção da estratégia de criação de valor pela empresa (Barney, 1991). Estratégias frágeis são passíveis de imitação e superação pelos competidores e, portanto, renderiam apenas uma vantagem temporária. No entanto, não há ilusões de que uma empresa encontre uma fonte de vantagem competitiva eterna (Barney, 1991). Mesmo a vantagem competitiva sustentada esvai-se com o tempo.

A busca pela vantagem competitiva sustentada sugere que os gestores invistam em recursos estratégicos de difícil replicação no médio-longo prazo. No entanto, a capacidade em reconhecer o potencial de criação de valor ex-ante depende do repertório de soluções e da experiência dos gestores (Amit \& Schoemaker, 1993). Já o sucesso na exploração de recursos e capacidades, quando percebido pelo mercado, passa a despertar movimentos de cópia e retaliação pelos competidores (Coff, 2010; Marcel, Barr, \& Duhaime, 2011). Portanto, a gestão dos resultados da criação de valor é tão importante quanto a própria criação de valor.

A importância da competência gerencial em reconhecer e desenvolver recursos e capacidades sugere que o estudo de vantagem competitiva deve compreender um período típico de gestão. Adicionalmente, é importante ponderar que o período de desenvolvimento e exploração de recursos e capacidades varia entre os setores industriais e depende do estágio de maturidade da empresa (Coff, 2010).

Como um construto latente, vantagem competitiva não pode ser observada diretamente, e, no tocante à temporalidade, os estudos sobre dinâmica competitiva entre empresas têm sido 
primordialmente focados na análise de variáveis de lucratividade (McNamara, Vaaler, \& Devers, 2003; Wiggins \& Ruefli, 2005). Essa deficiência no processo de mensuração não permite conclusões mais amplas, afinal, sendo o lucro uma medida de apropriação de valor, o estudo da persistência da lucratividade não permite concluir sobre vantagem competitiva. Ademais, é possível que vantagem competitiva não esteja inteiramente alinhada com a manutenção da lucratividade superior e vice-versa (Pacheco-de-Almeida, 2010). Entre outras, é preciso que a análise do desempenho seja expandida para abarcar os demais resultados das decisões estratégicas que não somente a lucratividade.

Como decorrência da discussão até aqui desenvolvida, uma empresa é considerada em vantagem competitiva quando cria mais valor que seus concorrentes, em um dado intervalo de tempo, considerando que:

. O valor criado é a diferença entre a disposição a pagar e o custo de oportunidade;

- Os concorrentes são aqueles que competem diretamente com a empresa; e

. O intervalo de tempo esteja adequado ao ciclo de desenvolvimento de recursos.

Essa definição integra os vários interesses de pesquisa em estratégia e dá à vantagem competitiva à capacidade de abranger um amplo intervalo de interesses. Como tal, deve servir como pedra fundamental para as construções teóricas e observações empíricas sobre vantagem competitiva.

Se as empresas com vantagem competitiva certamente criam mais valor, não é certo que elas possam se apropriar do valor criado. Esse tópico passa a ser tratado no processo de barganha e distribuição do valor, em que aparecem os resultados de desempenho, como vemos a seguir.

\section{Vantagem Competitiva e sua Relação com o Desempenho Financeiro}

Uma vez definida vantagem competitiva como uma criação de valor superior aos concorrentes, o objetivo desta seção é discutir como esse valor superior pode se manifestar no desempenho financeiro da empresa.

Vantagem competitiva está claramente posicionada como um antecedente do desempenho, que pode trazer diferentes resultados a depender da estratégia de apropriação de valor das empresas. Isso caracteriza vantagem competitiva como condição insuficiente para o desempenho financeiro, e, notadamente, para o lucro superior (Powell, 2001; Tang \& Liou, 2010).

O processo de distribuição do valor criado é moldado pela dinâmica da criação de valor e pela barganha entre a empresa e seus colaboradores e parceiros comerciais. Esta seção concentra-se nas consequências da distribuição do valor para o desempenho financeiro da empresa.

\section{A distribuição do valor: os processos de apropriação}

Valor criado não é igual a valor apropriado. Conforme aqui definido, os custos de oportunidade e disposição a pagar definem o valor criado, enquanto preço e custo definem a apropriação de valor criado. A parcela de valor entre a disposição a pagar e o preço, é, em princípio, apropriada pelos clientes como um excedente em relação ao preço que pagam. A parcela entre o preço e o custo é a parte diretamente apropriada pela empresa. E a parcela entre o custo da empresa e o custo de oportunidade dos fornecedores é apropriada pelos fornecedores. Todas essas três parcelas podem ter impactos sobre o desempenho financeiro da empresa, como desenvolvido no restante desta seção.

O processo de barganha e distribuição de valor não pode ser totalmente desvinculado das circunstâncias da criação de valor; no entanto, são processos distintos. Ao incorporar alguns aspectos comportamentais, os autores Brandenburger e Stuart (2007) sugerem uma combinação entre modelos 
de jogos cooperativos e não-cooperativos ao processo de barganha, nos chamados jogos Biformes. Dividindo o processo em dois tempos, considera-se o momento da criação de valor separadamente do de apropriação de valor, permitindo a combinação de resultados nos dois momentos (Chatain \& Zemsky, 2007). No entanto, os modelos têm ainda o desafio de lidar com a subjetividade da interpretação dos atores, com a variabilidade das situações e com pressupostos comportamentais (Brandenburger \& Stuart, 2007).

A assimetria de informação e de conhecimento entre os participantes influencia na capacidade de cada um em se apropriar do valor criado (Coff, 1999) e traz diferentes implicações ao longo do tempo: ao fundar uma empresa, empreendedores buscam modelos organizacionais mais propícios à apropriação de valor; no processo de desenvolvimento desta, os parceiros comerciais e gestores buscam arquitetar contratos mais favoráveis; já no estágio de consolidação, a rivalidade entre competidores faz com que os clientes possam acabar por apropriar mais valor (Coff, 2010).

Em todo o processo, a atividade do gestor é de grande importância, sendo esse um stakeholder interno com privilegiado acesso à informação (Coff \& Lee, 2003). É do gestor o papel de ajustar a função utilidade dos vários parceiros envolvidos, percebendo a utilidade marginal e o valor atribuído pelas partes. No processo é importante assegurar que a partilha de valor não obstrua processos de aliança e reciprocidade com os parceiros comerciais de interesse da empresa, uma vez que as parcerias são importantes na criação de valor (Bosse, Phillips, \& Harrison, 2009; Padula \& Dagnino, 2007).

\section{O excedente do cliente: preferência e crescimento}

A diferença entre a disposição a pagar e o preço, o excedente do cliente, representa o valor apropriado pelos clientes (Bowman \& Ambrosini, 2000; Priem, 2007). Embora seja um benefício direto para os clientes, essa parcela não deixa de ter efeito no desempenho da empresa. Se uma empresa oferece um excedente superior aos concorrentes, ela terá consequências positivas. Sendo o preço resultado de condições de demanda e competição, a interação da empresa com o mercado permite estratégias com diferentes os resultados (Figura 3).

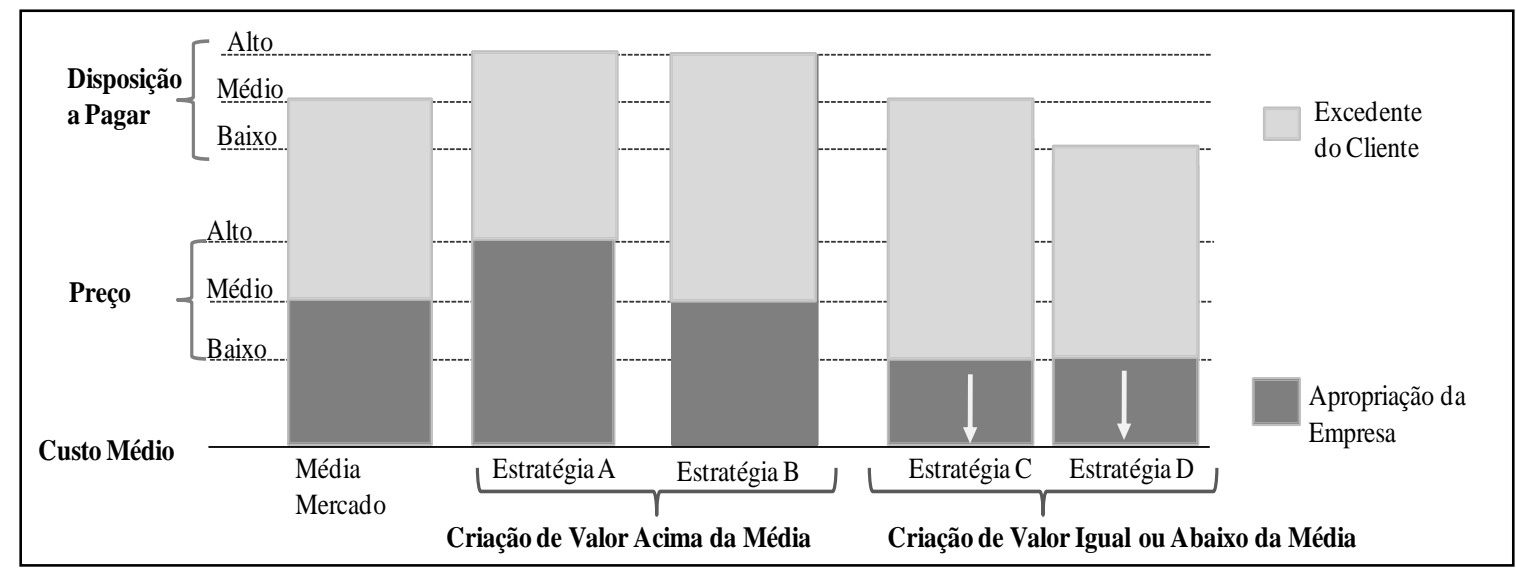

Figura 3. Estratégias para a Apropriação de Valor na Interação com Clientes.

Fonte: Adaptado de Bowman, C., \& Ambrosini, V. (2000). Value creation versus value capture: towards a coherent definition of value in strategy (p. 3). British Journal of Management, 11(1), 1-15. doi: 10.1111/1467-8551.00147

A empresa com vantagem competitiva pode explorar a assimetria entre a máxima disposição a pagar de seus produtos e a média do mercado, cobrando um prêmio de preço (Newbert, 2008; Porter, 1985). Essa estratégia de apropriação de valor (Figura 3 - Estratégia A) resultará em margens mais altas. Nota-se que a empresa que oferece maior valor para o cliente poderá ter insumos e condições diferenciadas de seus fornecedores. Sendo assim, a apuração de lucro econômico dependerá ainda da barganha com fornecedores e da definição o custo de oportunidade da empresa (Crook, Ketchen, Combs, \& Todd, 2008). 
Por outro lado, a empresa com vantagem competitiva pode escolher por manter a paridade de preços com seus concorrentes (Figura 3 - Estratégia B). Ao oferecer maior excedente para o cliente, essa empresa pode almejar tanto a proteção de seu mercado como a expansão de sua participação (Chatain, 2011; Newbert, 2008; Porter, 1985; Priem, 2007). Em ambos os casos, o excedente do cliente leva à preferência dos compradores, como uma recompensa pelo valor criado (Bowman \& Ambrosini, 2000; Chatain, 2011). Sendo assim, o excedente do cliente é uma parcela do valor não apropriada pela empresa, mas nem por isto irrelevante, pois também traz implicações para o desempenho.

A decisão de paridade de preço (Figura 3 - Estratégia B) pode se traduzir em uma estratégia de crescimento de mercado. $\mathrm{O}$ crescimento pode, eventualmente, levar a ganhos de escala que também se refletem na diminuição de custos e em maior lucratividade. No entanto, é importante considerar que o crescimento é uma forma de desempenho em si. O posicionamento de preço em relação à média do mercado determina tanto o potencial de crescimento como o nível de lucratividade, e os dois resultados de desempenho podem ser combinados ou individualmente favorecidos em diferentes momentos estratégicos. Entre outros, a estratégia de crescimento pode acelerar o acúmulo de conhecimento sobre a experiência de compra e uso do produto, e assim aumentar a utilidade percebida pelos clientes (Priem, 2007).

A capacidade de manobra dependerá do valor adicionado pela empresa. Em posição de vantagem competitiva, a empresa pode escolher estratégias que trazem um desempenho financeiro superior, seja lucratividade e/ou crescimento. Já empresas em paridade ou desvantagem não podem alcançar os mesmos resultados. Para crescer, empresas com paridade competitiva têm que aumentar o excedente do cliente e sacrificar sua lucratividade; para conseguir lucratividade acima da média, podem perder mercado (Figura 3 - Estratégias C e D).

A possibilidade de diferentes estratégias suscita a ideia de que há um ponto ótimo de equilíbrio entre lucratividade e crescimento. Isso ressalta a importância do apreçamento como um complexo efeito moderador na relação entre vantagem competitiva e o desempenho financeiro. Como principal responsável pelas decisões estratégicas, o gestor tem o dilema de lidar com as escolhas de maximização do lucro ou do crescimento, conhecendo o melhor momento para cada decisão (Cubbin \& Leech, 1986; Penrose, 1959; Slater, 1980).

Portanto, à empresa com vantagem competitiva são possíveis diferentes combinações, precificação, e, assim, diferentes resultados de desempenho. $\mathrm{O}$ excedente do cliente é função direta da definição do preço, mas já o lucro dependerá da negociação do custo, o que acontece no processo de barganha com fornecedores, gestores e empregados.

\section{O valor apropriado pela empresa: lucro e rentabilidade}

Determinada pelo intervalo entre preço e custo, esta parcela de valor se manifesta diretamente no lucro da empresa. A responsabilidade da empresa para com seus acionistas é tida como primordial, e é embasada na necessidade de remuneração do capital empregado (Brandenburger \& Stuart, 1996; Penrose, 1959). Como consequência desse entendimento, a proposta de maximização de valor é normalmente focada pela apuração do lucro, a parcela atribuída aos acionistas (Jensen, 2001).

Nessa lógica, boa parte dos modelos de barganha busca descrever a forma e os determinantes da apropriação de valor (Adegbesan, 2009; Brandenburger \& Stuart, 2007; Lippman \& Rumelt, 2003a; MacDonald \& Ryall, 2004). De um lado negociando pelo menor custo, a exemplo das grandes cadeias de varejo, do outro lado praticando prêmio de preço, a exemplo dos produtos superiores, a lucratividade passa a ser o principal objetivo da empresa. No entanto, nota-se que nem sempre a empresa conseguirá se apropriar de todo o valor criado e que, consequentemente, nem sempre a capacidade de criação de valor estará manifestada em lucratividade superior (Crook et al., 2008). É o caso de indústrias com forte rivalidade e produtos de demanda elástica, nos quais variações no preço 
podem resultar em perda de mercado. Nestes casos, mesmo com produtos superiores, a empresa pode não conseguir impor um prêmio de preço, tendo que deixar um maior excedente aos clientes.

Em outras situações, a criação de valor com baixa lucratividade é também uma forma de não chamar a atenção dos competidores e evitar a imitação e consequente erosão de desempenho (Coff, 2010). Ademais, na busca pela maximização da riqueza dos acionistas, outras medidas como consistência na trajetória e crescimento, capacidade de inovação e mesmo reputação, podem evidenciar melhor o valor da empresa no tempo (Richard et al., 2009).

\section{O benefício do fornecedor: benefícios defasados e ganhos operacionais}

A parcela entre o custo e o custo de oportunidade dos fornecedores fica, em princípio, com os fornecedores como um incentivo para o relacionamento, mas também pode, em algumas circunstâncias, trazer benefícios para a empresa focal. Tanto a literatura de estratégia, através da visão relacional (Dyer \& Singh, 1998), como a literatura de operações (Terpend, Tyler, Krause, \& Handfield, 2008) indicam mecanismos pelos quais relacionamentos com fornecedores podem provocar melhorias em desempenho operacional.

As circunstâncias de criação de valor se inserem na complexidade das relações contratuais que definem a empresa (Jensen \& Meckling, 1976), em que os recursos e capacidades são desenvolvidos em coespecialização e complementaridade. A complexidade do processo impede a delimitação clara dos direitos de propriedade das partes envolvidas, sendo que essas também têm percepções e expectativas diferentes sobre o valor dos recursos e as capacidades desenvolvidos. Esse cenário obscuro não favorece o processo de divisão de valor, mas traz consequências benéficas, como barreiras à imitação.

Na relação com fornecedores, custos de transação e oportunismo são frequentemente tidos como limites para o desenvolvimento de ativos específicos (Williamson, 1981). Na medida em que parcerias geram dependências, estas passam a ser vistas como possíveis entraves e potencial problema de holdup para a empresa. A não existência de instrumentos para controle do direito de propriedade impõem limites à apropriação do valor criado (Adegbesan \& Higgins, 2011). Por outro lado, a partilha desse valor, mantendo um excedente para o fornecedor, pode construir caminhos para a manutenção da vantagem competitiva. Isso porque alianças e parcerias são importantes no desenvolvimento de inovação e qualidade (Inkpen \& Tsang, 2005; Thomke \& Hippel, 2002) e podem ainda reduzir os custos de negociação e impor instrumentos de governança mais efetivos (Bosse et al., 2009; Dyer \& Singh, 1998). Assim sendo, vantagem competitiva pode gerar rendas relacionais (Dyer \& Singh, 1998) que se manifestam primordialmente no desempenho operacional da empresa. Os trabalhos de Dyer (1996, 1997), Dyer e Hatch (2006) sobre a indústria automobilística e a literatura de gestão estratégica de fornecedores e gestão da cadeia de suprimentos dão evidências desse efeito (Frohlich \& Westbrook, 2001). Embora a relação não seja direta, o desempenho operacional pode também trazer efeitos sobre o desempenho financeiro, seja na lucratividade, no crescimento ou até no valor de mercado da empresa (Combs, Crook, \& Shook, 2005). 


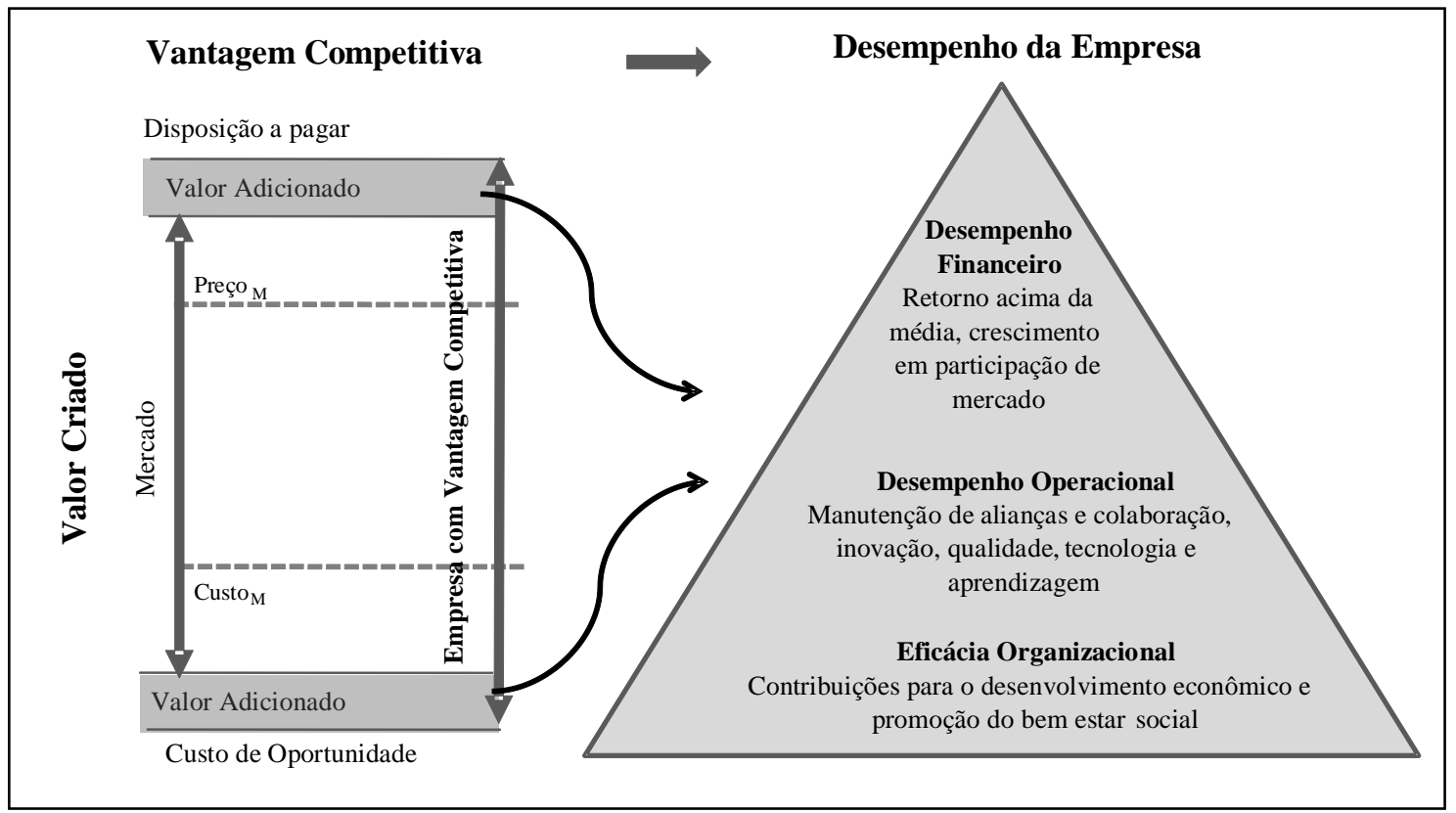

Figura 4. Relação entre Vantagem Competitiva e Desempenho.

Portanto, ao longo da cadeia, o processo de criação de valor, seguido pela partilha do valor criado, traz efeitos para as várias dimensões do desempenho organizacional (Figura 4), seja financeiro, operacional ou para a eficácia organizacional, organizational effectiveness (Venkatraman \& Ramanujam, 1986). Isso faz com que a vantagem competitiva, ou seja, a empresa que cria valor superior aos concorrentes, possa apresentar diferentes resultados de desempenho no tempo e até manter-se à frente sem ser notada.

\section{Conclusões}

O termo vantagem competitiva tem sido usado na área de estratégia de forma problemática, sem uma definição precisa, sendo muitas vezes confundido com desempenho superior (Powell, 2001). Percebe-se, porém, uma convergência da literatura mais recente no uso do conceito de valor criado pela empresa para definir vantagem competitiva (Coff, 2010; Peteraf \& Barney, 2003).

Este ensaio revisa de forma crítica a literatura de vantagem competitiva e propõe uma definição integrativa como criação de valor econômico superior aos concorrentes diretos. Além de definir com mais objetividade o que se entende por valor econômico, o artigo também discute os processos de criação e apropriação desse valor, e o desempenho financeiro como decorrente desta apropriação. Cria-se, dessa forma, uma lógica causal entre vantagem competitiva e desempenho financeiro.

O conceito de valor pode ser tratado de forma ampla, tomando-se a perspectiva de diferentes stakeholders e incluindo aspectos que afetam toda a sociedade. Seguindo a citada convergência na área de estratégica, a definição de vantagem competitiva proposta foca-se, contudo, na perspectiva do acionista, usando o valor econômico criado por uma empresa ao operar. Esse valor é a diferença entre a disposição a pagar dos clientes pelos produtos e serviços oferecidos pela empresa e o custo de oportunidade dos fornecedores dessa empresa (Brandenburger \& Stuart, 1994). O preço praticado é um valor menor que a disposição a pagar, e a diferença entre disposição a pagar e preço é o excedente do cliente, a parte do valor que a empresa cria por existir, mas deixa com seus clientes como motivação para transacionarem com ela. A empresa tem um custo econômico para produzir os seus bens e serviços, e a diferença entre o preço e o custo representa o valor apropriado diretamente pela empresa e determina o seu lucro. Já os fornecedores que vendem matérias primas e serviços à empresa, fazem-no porque veem nisso algo atrativo. Se essa empresa focal deixar de existir, eles terão 
que procurar uma segunda alternativa. A valorização da segunda melhor alternativa sob a perspectiva do fornecedor constitui o custo de oportunidade deste em relação à empresa focal. A diferença entre o custo da empresa e o custo de oportunidade dos fornecedores é uma parcela de valor que a empresa focal cria por existir e operar, mas que fica com os seus fornecedores como motivação para transacionarem com ela.

Essa definição de vantagem competitiva permite uma clara separação entre essa e o desempenho financeiro. Vantagem competitiva se refere à criação de valor. Desempenho financeiro decorre do processo de distribuição de valor entre as partes, o que não é um processo simples (Adegbesan \& Higgins, 2011) e resulta diferentes aspectos de desempenho. O benefício direto da empresa pode ser evidenciado por medidas de lucratividade ou rentabilidade, mas as outras duas parcelas também afetam o desempenho da empresa. O benefício do cliente pode, por exemplo, manifestar-se no crescimento. Se uma empresa deixa mais valor com seus clientes (uma oferta melhor a preços equivalentes), ela ganha mercado e tem um crescimento superior à média. $\mathrm{O}$ benefício do fornecedor é mais difícil de ser ligado diretamente ao desempenho financeiro, mas as literaturas da visão relacional e de operações sugerem que esses benefícios existem e talvez se manifestem na tendência no tempo por seu efeito em inovação e qualidade (Inkpen \& Tsang, 2005; Thomke \& Hippel, 2002), ou na regularidade do desempenho financeiro em seus múltiplos indicadores por contrapartidas sequenciais entre fornecedor e comprador (Dyer \& Hatch, 2006).

A proposta traz implicações para a academia e para a prática empresarial. Na perspectiva acadêmica, ela oferece uma interpretação mais integrativa de abordagens anteriores ou isoladas. É possível, por exemplo, generalizar o entendimento das estratégias genéricas de Porter (1985) de diferenciação e liderança de custos, como explicitado na Figura 3. O conceito também permite a integração de áreas que tratam das relações da empresa com seus fornecedores e clientes como Marketing e Operações com Estratégia. Em Marketing, ao segmentar o mercado e fazer ofertas mais adequadas a cada segmento, a empresa está aumentando a disposição a pagar média de seus clientes e, portanto, criando mais valor. Em Operações, ao desenvolver um portfólio adequado de fornecedores e desenvolver práticas colaborativas com alguns deles, a empresa pode conseguir vantagens de reduzir o seu custo ao longo do tempo (ou aumentar a disposição a pagar ao longo deste) e motivar o fornecedor, deixando para ele uma parcela do valor criado.

Uma das implicações mais relevantes, contudo, refere-se a como usar indicadores de desempenho financeiro para evidenciar a existência de vantagem competitiva. Medidas de lucratividade ou rentabilidade não são suficientes, pois capturam apenas uma parte do valor criado. Apesar disso, a grande maioria dos estudos em estratégia usa apenas medidas desse tipo, e muitas vezes apenas uma - o retorno sobre ativos. É fundamental incluir também a dimensão crescimento, e há evidências de que certos recursos podem afetar diferentemente lucratividade e crescimento (Cho \& Pucik, 2005).

Na perspectiva da prática empresarial, a definição de valor criado pode oferecer um guia simples para decisões gerenciais. Um exemplo disso é o relato de David Neelman em uma palestra a executivos (D. Neelman, comunicação pessoal, 04 de junho de 2009) de como decidiu pela colocação de um serviço de televisão no encosto dos assentos da companhia aérea Azul: "Pedi que calculassem o custo de um monitor, por assento e por viagem. Era de um dólar! Quanto os passageiros valorizariam esse benefício? A decisão é clara: vale muito mais do que um dólar”.

O tema Vantagem Competitiva está longe de ser esgotado, e várias lacunas e oportunidades ainda se oferecem para pesquisa. Como medir disposição a pagar e custo de oportunidade? Como medir o desempenho e relacioná-lo à vantagem competitiva? Como considerar o tempo? Como incluir o elemento futuro e preditivo e não apenas o passado? Como incluir o valor para outros stakeholders? Essas são algumas das perguntas de um tema apaixonante e aparentemente inesgotável.

\section{Artigo recebido em 27.05.2011. Aprovado em 03.04.2012.}




\section{Referências}

Adegbesan, J. A. (2009). On the origins of competitive advantage: strategic factor markets and heterogeneous resource complementarity. Academy of Management Review, 34(3), 463-475. doi: 10.5465/amr.2009.40632465

Adegbesan, J. A., \& Higgins, M. J. (2011). The intra-alliance division of value created through collaboration. Strategic Management Journal, 32(2), 187-211. doi: 10.1002/smj.872

Allen, M. G. (1978). Strategic planning with a competitive focus. The McKinsey Quarterly, 14(3), 213.

Amit, R., \& Schoemaker, P. J. H. (1993). Strategic assets and organizational rent. Strategic Management Journal, 14(1), 33-46. doi:10.2307/2486548

Ansoff, H. I. (1965). Corporate strategy; an analytic approach to business policy for growth and expansion. New York: McGraw-Hill.

Arend, R. J. (2003). Revisiting the logical and research considerations of competitive advantage. Strategic Management Journal, 24(3), 279-284. doi: 10.1002/smj.285

Barney, J. B. (1991). Firm resources and sustained competitive advantage. Journal of Management, $17(1), 99-120$.

Besanko, D., Dranove, D., Shanley, M., \& Schaefer, S. (1996). Economics of strategy. Hoboken: NJ: John Wiley \& Sons, Inc.

Blyler, M., \& Coff, R. W. (2003). Dynamic capabilities, social capital, and rent appropriation: ties that split pies. Strategic Management Journal, 24(7), 677-686. doi: 10.1002/smj.327

Bosse, D. A., Phillips, R. A., \& Harrison, J. S. (2009). Stakeholders, reciprocity, and firm performance. Strategic Management Journal, 30(4), 447-456. doi: 10.1002/smj.743

Bowman, C., \& Ambrosini, V. (2000). Value creation versus value capture: towards a coherent definition of value in strategy. British Journal of Management, 11(1), 1-15. doi: 10.1111/14678551.00147

Brandenburger, A., \& Nalebuff, B. (1996). Co-opetition. New York: Doubleday.

Brandenburger, A., \& Stuart, H. W. (1996). Value-based business strategy. Journal of Economics \& Management Strategy, 5(1), 5-24. doi: 10.1111/j.1430-9134.1996.00005.x

Brandenburger, A., \& Stuart, H. (2007). Biform games. Management Science, 53(4), 537-549. doi: $10.1287 / \mathrm{mnsc} .1060 .0591$

Buysse, K., \& Verbeke, A. (2003). Proactive environmental strategies: a stakeholder management perspective. Strategic Management Journal, 24(5), 453-470. doi: 10.1002/smj.299

Caves, R. E. (1984). Economic analysis and the quest for competitive advantage. American Economic Review, 74(2), 127-132. doi: 10.2307/1816342

Chamberlin, E. (1933). The theory of monopolistic competition. Cambridge: Harvard University Press.

Chatain, O. (2011). Value creation, competition, and performance in buyer-supplier relationships. Strategic Management Journal, 32(1), 76-102. doi: 10.1002/smj.864 
Chatain, O., \& Zemsky, P. (2007). The horizontal scope of the firm: organizational tradeoffs vs. buyer-supplier relationships. Management Science, 53(4), 550-565. doi: $10.1287 / \mathrm{mnsc} .1060 .0680$

Cho, H.-J., \& Pucik, V. (2005). Relationship between innovativeness, quality, growth, profitability, and market value. Strategic Management Journal, 26(6), 555-575. doi: 10.1002/smj.461

Coff, R. W. (1999). When competitive advantage doesn't lead to performance: the resource-based view and stakeholder bargaining power. Organization Science, 10(2), 119-133. doi: 10.1287/orsc.10.2.119

Coff, R. W. (2010). The coevolution of rent appropriation and capability development. Strategic Management Journal, 31(7), 711-733. doi: 10.1002/smj.844

Coff, R. W., \& Lee, P. M. (2003). Insider trading as a vehicle to appropriate rent from R\&D. Strategic Management Journal, 24(2), 183-190. doi: 10.1002/smj.270

Combs, J. G., Crook, T. R., \& Shook, C. L. (2005). The dimension of organizational performance and its implications for strategic management research. In D. J. Ketchen \& D. D. Bergh (Eds.), Research methodology in strategy and management (Vol. 1, pp. 259-286). San Diego: Elsevier.

Crook, T. R., Ketchen, D. J., Jr., Combs, J. G., \& Todd, S. Y. (2008). Strategic resources and performance: a meta-analysis. Strategic Management Journal, 29(11), 1141-1154. doi: $10.1002 / \mathrm{smj} .703$

Cubbin, J., \& Leech, D. (1986). Growth versus profit-maximization: a simultaneous-equations approach to testing the marris model. Managerial \& Decision Economics, 7(2), 123-131. doi: 10.1002/mde.4090070209

D'Aveni, R. A., Dagnino, G. B., \& Smith, K. G. (2010). The age of temporary advantage. Strategic Management Journal, 31(13), 1371-1385. doi: 10.1002/smj.897

Dagnino, G. B., \& Padula, G. (2002, maio). Coopetitiion strategy: a new kind of interfirm dynamics for value creation. Proceedings of the European Academy of Management Second Annual Conference, Stockholm, Sweden.

Davis, E., \& Kay, J. (1990). Assessing corporate performance. Business Strategy Review, 1(2), 1-16. doi: 10.1111/j.1467-8616.1990.tb00007.x

Drnevich, P. 1., Madsen, T., \& Newbert, S. (2010, September). Competitive advantage: theoretical challenges \& insights from value-price-cost approaches. Proceedings of the Academy of Management, Montreal, CA.

Dyer, J. H. (1996). Specialized supplier networks as a source of competitive advantage: evidence from the auto industry. Strategic Management Journal, 17(4), 271-291. doi: 10.1002/(SICI)10970266(199604)

Dyer, J. H. (1997). Effective interfirm collaboration: how firms minimize transaction costs and maximize transaction value. Strategic Management Journal, 18(7), 535-556. doi: 10.1002/(SICI)1097-0266(199708)

Dyer, J. H., \& Hatch, N. W. (2006). Relation-specific capabilities and barriers to knowledge transfers: creating advantage through network relationships. Strategic Management Journal, 27(8), 701719. doi:10.1002/smj.543

Dyer, J. H., \& Singh, H. (1998). The relational view: cooperative strategy and sources of interorganizational competitive advantage. Academy of Management Review, 23(4), 660-679. doi: $10.2307 / 259056$ 
Foss, K., \& Foss, N. J. (2005). Resources and transaction costs: how property rights economics furthers the resource-based view. Strategic Management Journal, 26(6), 541-553. doi: $10.1002 / \mathrm{smj} .465$

Foss, N. J., \& Knudsen, T. (2003). The resource-based tangle: towards a sustainable explanation of competitive advantage. Managerial \& Decision Economics, 24(4), 291-307. doi: $10.1002 /$ mde. 1122

Freeman, R. E. (1984). Strategic management: a stakeholder approach. Boston: Pitman.

Frohlich, M. T., \& Westbrook, R. (2001). Arcs of integration: an international study of supply chain strategies. Journal of Operations Management, 19(2), 185-200.

Ghemawat, P. (2002). Competition and business strategy in historical perspective. Business History Review, 76(1), 37-74. doi: 10.2307/4127751

Ghemawat, P., \& Rivkin, J. W. (2006). Creating competitive advantage. Boston: Harvard Business School Note.

Gluck, F. W., Kaufman, S. P., \& Walleck, A. S. (1980). Strategic management for competitive advantage. Harvard Business Review, 58(4), 2-16.

Grant, R. M. (2002). Corporate strategy: managing scope and strategy content. In A. Pettigrew, H. Thomas, \& R. Whittington (Eds.), Handbook of strategy and management (pp. 72-97). London: Sage Publications Ltd.

Hansen, M. H., Hoskisson, R. E., \& Barney, J. B. (2008). Competitive advantage in alliance governance: resolving the opportunism minimization-gain maximization paradox. Managerial \& Decision Economics, 29(2/3), 191-208. doi: 10.1002/mde.1394

Harrison, J. S., Bosse, D. A., \& Phillips, R. A. (2010). Managing for stakeholders, stakeholder utility functions, and competitive advantage. Strategic Management Journal, 31(1), 58-74.

Hayes, R. H., \& Wheelwright, S. C. (1984). Restoring our competitive edge. New York: Wiley.

Hillman, A. J., \& Keim, G. D. (2001). Shareholder value, stakeholder management, and social issues: what's the bottom line? Strategic Management Journal, 22(2), 125-139. doi: 10.2307/3094310

Hitt, M. A., Ireland, R. D., \& Hoskinsson, R. E. (1999). Strategic management. Cincinnati: South Western College.

Hofer, C. W., \& Schendel, D. (1978). Strategy formulation: analytical concepts. St. Paul, MN: West.

Hoopes, D. G., Madsen, T. L., \& Walker, G. (2003). Guest editors' introduction to the special issue: why is there a resource-based view? Toward a theory of competitive heterogeneity. Strategic Management Journal, 24(10), 889-902. doi: 10.1002/smj.356

Inkpen, A. C., \& Tsang, E. W. K. (2005). Social capital, networks, and knowledge transfer. Academy of Management Review, 30(1), 146-165.

Ito, N. C., Hayashi, P., Jr., Gimenez, F. A. P., \& Fensterseifer, J. E. (2012). Valor e vantagem competitiva: buscando definições, relações e repercussões. Revista de Administração Contemporânea, 16(2), 290-307. Recuperado de http://www.scielo.br/pdf/rac/v16n2/v16n2a08.pdf. doi: 10.1590/S1415-65552012000200008

Jensen, M. C. (2001). Value maximization, stakeholder theory, and the corporate objective function. European Financial Management, 7(3), 297-317. 
Jensen, M. C., \& Meckling, W. H. (1976). Theory of the firm: managerial behavior, agency costs and ownership structure. Journal of Financial Economics, 3(4), 305-360. doi: 10.2139/ssrn.94043

Krishna, A. (1991). Effect of dealing patterns on consumer perceptions of deal frequency and willingness to pay. Journal of Marketing Research, 28(4), 441-451. doi: 10.2307/3172784

Lepak, D. P., Smith, K. G., \& Taylor, M. S. (2007). Value creation and value capture: a multi-level perspective. Academy of Management Review, 32(1), 180-194. doi: 10.5465/amr.2007.23464011

Lindgreen, A., \& Wynstra, F. (2005). Value in business markets: what do we know? Where are we going? Industrial Marketing Management, 34(7), 732-748. doi: 10.1016/j.indmarman.2005.01.001

Lippman, S. A., \& Rumelt, R. P. (2003a). A bargaining perspective on resource advantage. Strategic Management Journal, 24(11), 1069-1086. doi: 10.1002/smj.345

Lippman, S. A., \& Rumelt, R. P. (2003b). The payments perspective: micro-foundations of resource analysis. Strategic Management Journal, 24(10), 903-927. doi: 10.1002/smj.346

Ma, H. (2000). Competitive advantage and firm performance. Competitiveness Review, 10(2), 15-32. doi: 10.1108/eb046396

MacDonald, G., \& Ryall, M. D. (2004). How do value creation and competition determine whether a firm appropriates value? Management Science, 50(10), 1319-1333. doi: $10.1287 / \mathrm{mnsc} .1030 .0152$

Marcel, J. J., Barr, P. S., \& Duhaime, I. M. (2011). The influence of executive cognition on competitive dynamics. Strategic Management Journal, 32(2), 115-138. doi: 10.1002/smj

McNamara, G., Vaaler, P. M., \& Devers, C. (2003). Same as it ever was: the search for evidence of increasing hypercompetition. Strategic Management Journal, 24(3), 261-278. doi: 10.1002/smj.295

Morrisson, J. R., \& Lee, J. G. (1979). The anatomy of strategic thinking. The McKinsey Quarterly, 15(3), 2-9.

Newbert, S. L. (2008). Value, rareness, competitive advantage, and performance: a conceptual-level empirical investigation of the resource-based view of the firm. Strategic Management Journal, 29(7), 745-768. doi: 10.1002/smj.686

Ohmae, K. (1978). Effective strategies for competitive success. The McKinsey Quarterly, 14(4), 5059.

Pacheco-de-Almeida, G. (2010). Erosion, time compression, and self-displacement of leaders in hypercompetitive environments. Strategic Management Journal, 31(13), 1498-1526. doi: $10.1002 / \mathrm{smj} .896$

Padula, G., \& Dagnino, G. B. (2007). Untangling the rise of coopetition. International Studies of Management \& Organization, 37(2), 32-52.

Penrose, E. (1959). The theory of the growth of the firm (3th ed.). Oxford, UK: Oxford University Press.

Peteraf, M. A. (1993). The cornerstones of competitive advantage: a resource-based view. Strategic Management Journal, 14(3), 179-191. doi: 10.1002/smj.4250140303 
Peteraf, M. A., \& Barney, J. B. (2003). Unraveling the resource-based tangle. Managerial and Decision Economics, 24(4), 309-323. doi: 10.1002/mde.1126

Porter, M. E. (1985). Competitive advantage: creating and sustaining superior performance. New York: Free Press, Collier Macmillan.

Powell, T. C. (2001). Competitive advantage: logical and philosophical considerations. Strategic Management Journal, 22(9), 875-888. doi: 10.1002/smj.173

Priem, R. L. (2007). A consumer perspective on value creation. Academy of Management Review, 32(1), 219-235. doi: 10.5465/AMR.2007.23464055

Reed, R., \& DeFillippi, R. J. (1990). Causal ambiguity, barriers to imitation, and sustainable competitive advantage. Academy of Management Review, 15(1), 88-102. doi: 10.2307/258107

Richard, P. J., Devinney, T. M., Yip, G. S., \& Johnson, G. (2009). Measuring organizational performance: towards methodological best practice. Journal of Management, 35(3), 718-804. doi: $10.1177 / 0149206308330560$

Rumelt, R. P. (2003). What in the world is competitive advantage? [Policy Working Paper 2003-105]. Harry \& Elsa Kunin professor of Business \& Society the Anderson School at UCLA. Recuperado http://www.anderson.ucla.edu/faculty/dick.rumelt/Docs/Papers/WhatisCA_03.pdf

Slater, M. (1980). The managerial limitation to a firm's rate of growth. The Economic Journal, 90(359), 520-528.

Peteraf, M. (1981). Competitive advantage: the cornerstone of strategic thinking. Journal of Business Strategy, 1(4), 15-25. doi: 10.1108/eb038908

Tang, Y.-C., \& Liou, F.-M. (2010). Does firm performance reveal its own causes? The role of Bayesian inference. Strategic Management Journal, 31(1), 39-57. doi: 10.1002/smj.799

Teece, D. J., Pisano, G., \& Shuen, A. (1997). Dynamic capabilities and strategic management. Strategic Management Journal, 18(7), 509-533. doi: 10.1002/(SICI)10970266(199708)18:7<509::AID-SMJ882>3.0.CO;2-Z

Terpend, R., Tyler, B. B., Krause, D. R., \& Handfield, R. B. (2008). Buyer-supplier relationships: derived value over two decades. Journal of Supply Chain Management, 44(2), 28-55. doi: 10.1111/j.1745-493X.2008.00053.x

Thomke, S., \& Hippel, E. von (2002). Customers as innovators: a new way to create value. Harvard Business Review, 80(4), 74-81.

Vasconcelos, F. C., \& Brito, L. A. L. (2004). Vantagem competitiva: o construto e a métrica. Revista de Administração de Empresas, 44(2), 70-82. doi: 10.1590/S0034-75902004000200006

Venkatraman, N., \& Ramanujam, V. (1986). Measurement of business performance in strategy research: a comparison of approaches. Academy of Management Review, 11(4), 801-814. doi: $10.2307 / 258398$

Wertenbroch, K., \& Skiera, B. (2002). Measuring consumers' willingness to pay at the point of purchase. Journal of Marketing Research, 39(2), 228-241. doi: 10.1509/jmkr.39.2.228.19086

Wiggins, R. R., \& Ruefli, T. W. (2005). Schumpter's ghost: is hypercompetition making the best of times shorter? Strategic Management Journal, 26(10), 887-911. doi: 10.1002/smj.492 
Williamson, O. E. (1981). The economics of organizations: the transaction cost approach. American Journal of Sociology, 87(3), 548-577.

Williamson, O. E. (1991). Strategizing, economizing, and economic organization. Strategic Management Journal, 12(S2), 75-94. doi: 10.1002/smj.4250121007

Wood, L. M. (1996). Added value: marketing basics? Journal of Marketing Management, 12(8), 735755. doi: 10.1080/0267257X.1996.9964450 